\title{
La terapia enzimatica sostitutiva nella malattia di Fabry
}

\author{
Letizia Roggero, Sara Auricchio e Federico Pieruzzi
}

\begin{abstract}
Enzyme Replacement Therapy for Fabry Disease

Anderson-Fabry disease (FD) is a X-linked lysosomal storage disorder, which involves glycosphingolipids metabolism. Specific treatment for FD has been available in the last two decades, after the development and commercialization of recombinant human alfa-galactosidase A. Since then enzyme replacement therapy (ERT) has changed the natural history of the disease. Two different enzymatic formulations are available: agalsidase alfa and agalsidase beta at different dosages. The safety and efficacy profiles are similar. ERT induces Gb3 deposits reduction in renal and cardiac biopsies, improves quality of life, reduces pain and Gl symptoms, decreases left ventricular mass and slows down renal function decline. In case of organ involvement, clinical evidence confirms the need to treat all patients with enzyme therapy, both male and female. In all other clinical settings, the decision to start ERT is controversial, because of the extremely variable clinical manifestations of FD. However, data suggest a greater response to ERT if started as early as possible in any patients. Timely treatment appears to be effective in stabilizing and possibly delaying FD progression. ERT infusion reactions due to allergic hypersensitivity or IgG antibody development could occur but can be easily managed. In-hospital and at home infusions are possible. The wide genetic and phenotypic heterogeneity observed in all FD patients requires a tailored approach to treatment options. Patients should be referred to an expert multidisciplinary team for the long term management of this challenging disease.
\end{abstract}

\section{Keywords}

Fabry disease, lysosomal storage disease, alpha-galactosidase, enzyme replacement therapy, algasidase.

\section{Introduzione}

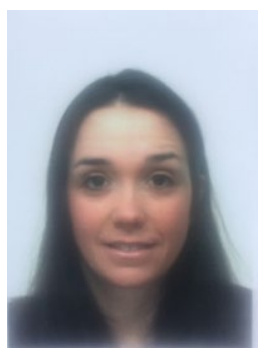

La malattia di Anderson-Fabry (MF) è un disordine da accumulo lisosomiale ad ereditarietà $\mathrm{X}$-linked, che coinvolge il metabolismo dei glicosfingolipidi, ed è causata dal deficit totale o parziale dell'enzima alfa-galattosidasi A codificato dal gene GLA. La carenza dell'attività dell'enzima conduce a un progressivo

Dottoressa Letizia Roggero per la MF inizia negli anni '70, quando comparvero in letteratura i primi tentativi di sostituire l'enzima mancante usando $\alpha$-galattosidasi A umana, dimostrando la potenziale utilità della terapia enzimatica sostitutiva. ${ }^{3}$ Negli anni ' 80 e '90, l'isolamento e il sequenziamento del cDNA codificante a-galattosidasi A umana ${ }^{4}$, la produzione di enzima umano ricombinante in cellule di ovaio di criceto cinese $(\mathrm{CHO})^{5}$, e lo sviluppo di un modello animale di MF fornirono gli strumenti per approfondire l'efficacia di questo trattamento. I primi studi preclinici hanno

Clinica Nefrologica, ASST- Monza, Scuola di Medicina e Chirurgia, Università degli Studi di Milano-Bicocca, Italy

\section{Corrispondenza:}

Dott. Federico Pieruzzi, Direttore f.f. S.C. Nefrologia ASST Monza, Clinica Nefrologica, Dipartimento di Medicina e Chirurgia, Università degli Studi di Milano-Bicocca, Ospedale San Gerardo, Via Pergolesi 33, Milan, Monza (MB) 20900, Italy.

E-mail: federico.pieruzzi@unimib.it 
dimostrato che la terapia enzimatica poteva ridurre il $\mathrm{Gb} 3$ accumulato, sia nel plasma che nei tessuti interessati (rene, milza e cuore), in modo dose-dipendente. ${ }^{6}$ Pertanto nel corso degli anni, le innovazioni delle biotecnologie moderne hanno reso possibile lo sviluppo e la commercializzazione di preparazioni di alfa-galattosidasi A umane ricombinanti, utilizzabili per il trattamento specifico della MF. Dal 2001 sono disponibili in prontuario farmaceutico due differenti formulazioni enzimatiche, agalsidasi alfa ed agalsidasi beta. ${ }^{7}$

\section{Terapia enzimatica sostitutiva: agalsidasi alfa e agalsidasi beta}

Agalsidasi alfa (Replagal, Shire, Cambridge, MA, USA), catalizza l'idrolisi di Gb3, separando il residuo terminale di galattosio dalla molecola. Viene prodotta, con preparazioni di ingegneria genetica, in linee cellulari umane, per ottenere un enzima costituito da un profilo di glicosilazione umano in grado di modificare la captazione dei recettori per il mannosio 6 fosfato sulla superficie delle cellule. La dose di $0,2 \mathrm{mg} / \mathrm{kg}$ ogni due settimane, somministrata per via endovenosa, è stata definita in grado di saturare la capacità dei recettori per il mannosio 6 fosfato. I dati degli studi clinici di registrazione suggeriscono che, per ottenere un adeguato volume di distribuzione ed una corretta farmacodinamica, la dose deve essere quantomeno pari a $0,1 \mathrm{mg} / \mathrm{kg}$, somministrato una volta ogni 2 settimane come infusione endovenosa. ${ }^{8}$

Agalsidasi beta (Fabrazyme, Genzyme Corp, Cambridge, $M A, U S A$ ), invece, è una forma ricombinante di $\alpha$-galattosidasi A umana ed è prodotta tramite tecnologia del DNA ricombinante da campioni cellulari di ovaio di criceto cinese (CHO). La sequenza di aminoacidi della forma ricombinante è identica alla forma naturale della $\alpha$-galattosidasi A. La dose suggerita di agalsidasi è di $1 \mathrm{mg} / \mathrm{kg}$, somministrato una volta ogni 2 settimane come infusione endovenosa. ${ }^{9}$

Agalsidasi alfa ha ottenuto l'autorizzazione della European Medicines Agency (EMA), mentre l'agalsidasi beta ha ricevuto l'autorizzazione sia da parte dell'EMA che da parte della Food and Drug Administration (FDA). ${ }^{10} \mathrm{La}$ FDA, per autorizzare l'approvazione di agalsidasi alfa negli Stati Uniti, ha richiesto ulteriori studi clinici controllati sul miglioramento del dolore e della funzione renale. ${ }^{10}$

\section{Terapia enzimatica sostitutiva: indicazioni}

Nonostante l'assenza di Linee Guida Internazionali sulla gestione della ERT nei pazienti con MF, le evidenze oggi disponibili concordano che vi è la necessità di iniziare la terapia enzimatica in tutti i pazienti, maschi e femmine, con segni e sintomi d'interessamento d'organo.

A causa delle variabili manifestazioni cliniche della malattia, se e quando iniziare l'ERT è un argomento controverso. Il panel di esperti europei ha dato alcune indicazioni per aiutare i clinici ad identificare i pazienti meritevoli di trattamento. ${ }^{11}$

Innanzitutto l'inizio dell'ERT richiede una diagnosi completamente certificata di MF, ovvero la conferma di malattia mediante esame genetico, attività enzimatica basso/assente e livelli elevati di Gb3 nel sangue.

La classe fenotipica ed il grado di coinvolgimento clinico della MF definiscono l'atteggiamento terapeutico.

Nei pazienti maschi con malattia classica di Fabry diagnosticata in età pediatrica, l'ERT dovrebbe essere iniziata nel corso dell'infanzia, in particolare in caso di insorgenza di sintomi correlati alla MF. ${ }^{12}$ Negli adulti maschi con mutazione classica l'ERT dovrebbe essere considerata in tutti i pazienti, qualsiasi sia la presentazione della malattia. Nelle pazienti adulte donne sintomatiche, con mutazione classica, l'ERT dovrebbe essere presa in considerazione in presenza di segni e sintomi che suggeriscono un chiaro coinvolgimento anche subclinico della malattia. Nelle donne asintomatiche con mutazione classica l'ERT è suggerita quando ci sia un'evidenza (laboratoristica, istologica o all'imaging) di danno renale, cardiaco o neurologico. Nei pazienti adulti maschi e femmine con mutazioni di Fabry ad insorgenza tardiva (forme late-onset, o varianti di significato incerto) l'ERT deve essere considerata una volta che vi è evidenza biochimica, istologica o di imaging a livello degli organi target, attribuibile alla MF. La valutazione della presenza dei depositi di $\mathrm{Gb} 3$ a livello tissutale nei pazienti con difficile interpretazione del genotipofenotipo, nelle mutazione non descritte e nei polimorfismi, può guidare la decisione riguardante l'indicazione o meno all'ERT. ${ }^{13-15}$

\section{Terapia enzimatica sostitutiva: risultati}

L'ERT è stata la prima terapia specifica che ha cambiato la storia naturale della MF. Diversi studi hanno confrontato l'attività specifica e la composizione biochimica dei due prodotti dimostrando che gli enzimi hanno essenzialmente le stesse attività e proprietà cinetiche, sebbene Fabrazyme abbia un elevato contenuto di mannosio-6-fosfato e quindi abbia una maggiore captazione a livello renale e cardiaco. Gli studi di comparazione tra le due formulazioni sono pochi, tuttavia quelli disponibili hanno suggerito che l'uso dei due farmaci è equivalente e conduce ad effetti clinici sovrapponibili. ${ }^{16-17}$ In particolare, in uno studio randomizzato controllato è stata confrontata l'efficacia e la tollerabilità dei due preparati. Trentaquattro pazienti affetti da MF sono stati trattati con agalsidasi alfa o agalsidasi beta alla stessa dose di $0,2 \mathrm{mg} / \mathrm{kg}$ ogni 15 giorni senza riscontrare sostanziali differenze cliniche: la variazione della massa ventricolare sinistra dopo 12 e 24 mesi, della velocità di filtrazione glomerulare, del dolore, dei livelli plasmatici ed urinari di 
GL-3 e la comparsa di anticorpi anti-agalsidasi erano sovrapponibili. ${ }^{18}$

La sicurezza e l'efficacia di Fabrazyme è stata valutata in due studi clinici multicentrici, multinazionali, randomizzati, in doppio cieco, controllati con placebo che hanno coinvolto rispettivamente 58 e 82 pazienti. ${ }^{19-20} \mathrm{Il}$ tasso degli eventi clinici è stato notevolmente inferiore nel caso dei pazienti trattati con Fabrazyme rispetto ai pazienti in terapia con placebo. L'efficacia dell'ERT con Fabrazyme nella stabilizzazione della malattia renale, il miglioramento del coinvolgimento cardiaco e la diminuzione del dolore alle estremità e delle manifestazioni gastrointestinali sono stati riportati in diversi studi. ${ }^{10,20}$

La sicurezza e l'efficacia di Replagal sono state valutate in due studi randomizzati in doppio cieco, placebocontrollati; 40 pazienti affetti da MF sono stati arruolati, a questi ultimi è stata somministrata la dose consigliata di $\operatorname{Replagal}(0,2 \mathrm{mg} / \mathrm{kg})$. Dopo $6 \mathrm{mesi}$ di terapia si è registrata una significativa riduzione del dolore con conseguente riduzione dell'abuso di analgesici. Dopo 6 mesi di trattamento la biopsia renale dei pazienti in terapia con Replagal ha mostrato un incremento dei glomeruli normali rispetto ai glomeruli sani del gruppo placebo. Il trattamento con Replagal induce la riduzione dei depositi di Gb3 nel plasma e nel sedimento urinario, nelle biopsie renali e cardiache. L'uso di Replagal sembra essere associato al miglioramento della contrattilità del miocardio, e alla diminuzione dello spessore del setto interventricolare. ${ }^{21-22}$

I dati clinici ottenuti finora, suggeriscono una maggior risposta al trattamento, se iniziato il più precocemente possibile, in giovane età. Il trattamento tempestivo, prima dell'insorgenza del danno d'organo conclamato a livello renale, cardiaco e neurologico, sembra essere efficace nel mitigare la progressione della malattia. Ad esempio, i pazienti che hanno iniziato il trattamento con beta agalsidasi prima dei 30 anni hanno presentato un declino statisticamente significativo della LVM. Inoltre evidenze ecocardiografiche e RMN confermano la riduzione dell'ipertrofia ventricolare, con un conseguente miglioramento della funzionalità cardiaca. L'inizio del trattamento prima dello sviluppo di glomerulosclerosi e prima dell'instaurarsi della proteinuria potrebbe prevenire future patologie renali. ${ }^{23}$

Nel complesso è dimostrato che l'ERT aumenta l'aspettativa di vita riducendo gli eventi clinici maggiori e migliora la qualità di vita dei pazienti, riducendo sensibilmente il dolore neuropatico ed i sintomi gastrointestinali. ${ }^{22}$

\section{Terapia enzimatica sostitutiva: vantaggi e svantaggi}

L'ERT viene somministrata a cadenza bisettimanale, mediante un'infusione endovenosa di durata variabile a seconda del dosaggio. Reazioni perinfusionali da ipersensibilità di tipo allergico, quali febbre e brivido, sono possibili, ma facilmente controllabili grazie alla modulazione della velocità di infusione e alla premedicazione con antistaminici, paracetamolo, ibuprofene e/o corticosteroidi. ${ }^{9,19}$ La formazione di anticorpi $\operatorname{IgG}$ verso gli enzimi infusi è relativamente comune soprattutto eni maschicon fenotipo classico ed è stata riportata con entrambe le forme di agalsidasi. Nella maggior parte dei pazienti normalmente lo sviluppo di anticorpi $\operatorname{IgG}$ avviene entro 3 mesi dalla prima infusione sebbene non sia chiaro se determinano un riduzione di efficacia della ERT. E' raccomandato il monitoraggio periodico degli anticorpi anti-agalsidasi dell'immunoglobulina $\mathrm{G}(\mathrm{IgG})$ del paziente. ${ }^{13,23} \mathrm{La}$ somministrazione endovenosa è inizialmente intraospedaliera. Nei pazienti ritenuti idonei vi è la possibilità di proseguire la terapia al proprio domicilio, laddove è stata ottenuta preventiva autorizzazione. L'infusione domiciliare, eseguita sotto la supervisione di personale qualificato, ha permesso di ridurre i costi e di migliorare indubbiamente la qualità di vita dei pazienti trattati, poiché dona ai malati una maggiore autonomia nella gestione della propria malattia.

\section{Conclusioni}

L'eterogeneità clinica della MF impone un approccio individualizzato all'assistenza del singolo paziente che tenga conto globalmente delle sue caratteristiche genetiche, fenotipiche e cliniche. L'ERT, disponibile dal 2001, è stato il primo trattamento specifico per la MF, fornendo una cura a migliaia di pazienti fino ad allora considerati non trattabili. Vi sono prove crescenti del fatto che la risposta clinica al trattamento migliori con l'inizio precoce e tempestivo della ERT. Data la complessità della malattia, la gestione a lungo termine di questi pazienti MF dovrebbe essere affidata ad un team multidisciplinare in grado di seguire la progressione della malattia in tutte le sue forme, avvalendosi anche di appropriate terapie aggiuntive.

\section{Dichiarazione di assenza di conflitto di interessi}

Gli Autori dichiarano di non aver conflitti di interessi.

\section{Finanziamenti}

Gli Autori dichiarano di non aver ricevuto finanziamenti specifici da qualsiasi ente nel settore pubblico, privato o senza fini di lucro.

\section{Bibliografia}

1 Pisani A, Visciano B, Imbriaco M, Di Nuzzi A, Mancini A, Marchetiello C and Riccio E. The Kidney in Fabry's disease. Clinical Genetics 2014; 86(4).

2 Rombach SM, Smid BE, Linthorst GE, Dijkgraaf MG and Hollak CE. Natural course of Fabry disease and the effectiveness of enzyme replacement therapy: a systematic review and meta-analysis: effectiveness of ERT in different disease stages. Journal of Inherited Metabolism Disease 2014; 37:341-352.

3 Roscoe PD, Brady O, Tallman JF, Johnson WG, Gal AE, Leahy WR, Quirk JM and Dekaban AS. Replacement 
therapy for inherited enzyme deficiency - Use of purified ceramidetrihexosidase in Fabry's ddisease. The New England Journal of Medicine 1973.

4 Bishop DF, Calhount DH, Bernstein HS, Hantzopoulost $\mathrm{P}$, Quinnt $\mathrm{M}$ and Desnick RJ. Human a-galactosidase A: Nucleotide sequence of a cDNA clone encoding the mature enzyme. Journal of Genetics 1986; 83(July):4859-4863.

5 Ioannou YA, Zeidner KM, Grace ME and Desnick RJ. Human alpha-galactosidase A: glycosylation site 3 is essential for enzyme solubility. Biochemical Journal 1998; 797:789-797.

6 Lee K, Jin X, Zhang K, Copertino L, Andrews L, Bakermalcolm J, Geagan L, Qiu H, Seiger K, Barngrover D, Mcpherson JM and Edmunds T. A biochemical and pharmacological comparison of enzyme replacement therapies for the glycolipid storage disorder Fabry disease. Glycobiology 2003; 13(4).

7 Alegra T, Vairo F, de Souza MV, Krug BC and Schwartz IV. Enzyme replacement therapy for Fabry disease: A systematic review and meta-analysis. Genetics and Molecular Biology 2012; 35:947-954.

8 European Medicine Agency: Replagal ${ }$ SmPC 2015; http://www.ema.europa.eu/docs/en_GB/document_library/ EPAR_Summary_for_the_public/human/000369/ WC500053606.pdf

9 European Medicine Agency: Fabrazyme ${ }^{\circledR}$ SmPC 2013; http:// www.ema.europa.eu/docs/en_GB/document_library/EPAR Summary_for_the_public/human/000370/WC500020541.pdf

10 Desnick RJ. Enzyme replacement therapy for Fabry disease: lessons from two alpha-galactosidase A orphan products and one FDA approval. Expert Opinion on Biological Therapy 2004.

11 Wanner C, Arad M, Baron R, Burlina A, Elliott PM, FeldtRasmussen U, Fomin VV, Germain DP, Hughes DA, Jovanovic A, Kantola I, Linhart A, Mignani R, Monserrat L, Namdar M, Nowak A, Oliveira JP, Ortiz A, Pieroni M, Spada M, Tylki-Szymańska A, Tøndel C, Viana-Baptista M, Weidemann F and Hilz MJ. European expert consensus statement on therapeutic goals in Fabry disease. Molecular Genetics and Metabolism 2018; 124(3).

12 Spada M, Baron R, Elliott PM, Falissard B, Hilz MJ, Monserrat L, Tøndel C, Tylki-Szymańska A, Wanner C and Germain DP. The effect of enzyme replacement therapy on clinical outcomes in pediatric patients with Fabry disease - A sistematic literature review by a European panel of experts. Molecular Genetics and Metabolism 2018; April.

13 Ortiz A, Germain DP, Desnick RJ, Politei J, Mauer M, Burlina A, Eng C, Hopkin RJ, Laney D, Waldek S, Wallace E, Weidemann F and Wilcox WR. Fabry disease revisited: Management and treatment recommendations for adult patients. Molecular Genetics and Metabolism 2018; 123(February):416-427.

14 Germain DP and Elliott PM. The effect of enzyme replacement therapy on clinical outcomes in male patients with Fabry disease - A systematic literature review by a European panel of experts. Molecular Genetics and Metabolism 2019; January.

15 Germain DP, Arad M, Burlina A, Elliott PM, Falissard B, Feldt-Rasmussen U6, Hilz MJ7, Hughes DA, Ortiz A, Wanner C, Weidemann F and Spada M. The effect of enzyme replacement therapy on clinical outcomes in female patients with Fabry disease - A systematic literature review by a European panel of experts. Molecular Genetics and Metabolism 2018; September.

16 Sirrs S, Clarke JT, Bichet DG, Casey R, Lemoine K, Flowerdew G, Sinasac DS and West ML. Baseline characteristics of patients enrolled in the Canadian Fabry Disease Initiative. Molecular Genetics and Metabolism Reports 2010; 99(4):367-373.

17 Limgala RP, Jennelle T, Plassmeyer M, Boutin M and Lavoie P. Altered immune phenotypes in subjects with Fabry disease and responses to switching from agalsidase alfa to agalsidase beta. American Journal of Translation Research 2019; 11(3):1683-1696.

18 Vedder AC, Linthorst GE, Houge G, Groener JEM, Ormel EE, Bouma BJ and Aerts JMFG. Treatment of Fabry disease: outcome of a comparative trial with agalsidase alfa or beta at a dose of $0.2 \mathrm{mg} / \mathrm{kg}$. PloS One 2007; 7 .

19 Eng CM and Guffon N. Safety and efficacy of recombinant human alpha-galactosidase A replacement therapy in Fabry's disease. The New England Journal of Medicine 2001; 345(1):9-16.

20 Wilcox WR, Banikazemi M, Guffon N, Waldek S, Lee P, Linthorst GE, Desnick RJ and Germain DP International Fabry Disease Study Group. Long-term safety and efficacy of enzyme replacement therapy for Fabry disease. American Journal of Human Genetics 2004.

21 Sasa H, Nagao M and Kino K. Safety and effectiveness of enzyme replacement therapy with agalsidase alfa in patients with Fabry disease: Post-marketing surveillance in Japan. Molecular Genetics and Metabolism 2019; April.

22 Schaefer RM, Tylki-Szymańska A and Hilz MJ. Enzyme replacement therapy for Fabry disease: a systematic review of available evidence. Drugs 2009; 69(16).

23 Arends M, Biegstraaten M, Hughes DA, Mehta A, Vaz M, Elliott M, Oder D, Watkinson OT and Wanner C. Retrospective study of long-term outcomes of enzyme replacement therapy in Fabry disease: Analysis of prognostic factors. PloS One 2017. 